\title{
Absorption of light in positron and electron states in quasi-zero-dimensional nanosystems
}

\author{
Sergey I. Pokutnyi, Petro P. Gorbyk \\ 17 Naumov Str., A.A.Chujko Institute of surface chemistry NASU, UA - 03164, Kyiv, Ukraine
}

Email address:

Pokutnyi_Sergey@inbox.ru(S. I. Pokutnyi)

\section{To cite this article:}

Sergey I. Pokutnyi, Petro P. Gorbyk. Absorption of Light in Positron and Electron States in Quasi-Zero-Dimensional Nanosystems. Optics. Vol. 2, No. 4, 2013, pp. 47-50. doi: 10.11648/j.optics.20130204.11

\begin{abstract}
A theory is developed for the interaction of an electromagnetic field with one-particle quantum-confined states of positron and electron in spherical nanovoids of metals and semiconductors. In the framework of dipole approximation it is shown that the oscillator forces and dipole moments for the transitions involving one-particle states in nanovoids are rather large, exceeding the corresponding typical parameters of bulk metal and semiconductor materials. It is established that giant optical absorption cross sections in the quasi-zero-dimensional systems to enable to use these nanosystems as new efficient absorbing nanomaterials for infra-red radiation.
\end{abstract}

Keywords: Coulomb States, Positron and Electron States, Forces of Oscillators, Dipole Moments, Nanovoids, Absorption of Light

\section{Introduction}

At the last decade a lot attention has been paid to find the strong-absorbing nano-materials with next assignment to build the artificial analogue of the photosynthesis nanostructures [1-4]. Therefore the investigations of the optical and electrooptics properties of the strong-absorbing nano-materials are actual. It is important to note, as it is shown at the present work, the quasizerodimensional nanosystems, consisted from the dielectric nanoparticle (nanoporous) of the spherical shape and immersed in the matrix of the metal and semiconductor, are strongly absorbing nanomaterials specially for the infrared radiation.

The optical [1-6] and electro-optical [7, 8] properties of quasi-zero-dimensional structures, consisted from semiconductor, dielectric and metal nanoparticles of spherical form with radius $a=1-10 \mathrm{~nm}$, synthesized in semiconductor, dielectric and metal matrixes are under intensive investigations at present time. The such linear sizes $a$ are consistent with de Broglie wave length of the quasi-particle (electron, positron) or (and) with Bohr radius of them. As result the phenomena of the space quantization of the quasi-particle plays significant role in the optical [1-6] and electro-optical $[7,8]$ processes in the such nanosystems.

In the experimental works $[15,16]$, by the method of the positron annihilation, in the nano-crystal of the metal and semiconductor, it was found the vacancy spherical nanoporous with the radiuses $a=15-20 \mathrm{~nm}$.

The investigations caused by the fact that such quasizero-dimensional structures serve as new perspective nanomaterials for design the new elements of nonlinear optoelectronics (especially as active domain of injection semi-conductor nanolaser [8] as well as new strongly absorbing nanomaterials [9-11]).

In present work in framework of dipole approach it is shown, that the forces of oscillators of transitions as well as dipole moments of transitions for one-particle positron and electron states, originated in spherical nanoporous of metals and semi-conductors, reach huge values, significantly higher (on two orders) the typical values corresponding values for metal and semi-conductors. It is found that the giant value of cross section of absorbing of light in considered nanosystems to enable to use such nanosystems as strongly absorbing nanomaterials for infrared raiation.

\section{The Forces of Oscillators and Dipole Moments of Transitions in Nanosystems}

Consider the simple model of quasi-zero-dimensional 
system: neutral spherical dielectric nanoparticle radius $a$ with permittivity $\varepsilon_{2}$ surrounded by medium with permittivity $\varepsilon_{1} \quad$ (the relative permittivity $\mathcal{E}=\left(\varepsilon_{2} / \varepsilon_{1}\right)<<1$ ). A quasi-particle (positron, electron or hole) moves in the volume of the nanoparticle with effective mass $m_{p(e, h)}[1-4]$. As the all principle sizes of

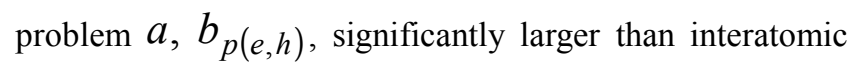
distance $a_{0}$, the motion of quasi-particle in the nanoparticle can be considered in the approach of effective mass [1-4]. Here the value

$$
b_{p(e, h)}=6 \beta^{-1} a_{p(e, h)}
$$

is the average distance of quasi-particle from interface in ground state, parameter

$$
\beta=\left(\varepsilon_{1}-\varepsilon_{2}\right) /\left(\varepsilon_{1}+\varepsilon_{2}\right)
$$

$a_{p(e, h)}=\left(\varepsilon_{2} \hbar^{2} / m_{p(e, h)} e^{2}\right)$ - Bohr radius of quasiparticle in the medium with permittivity $\varepsilon_{2}$.

In [12] the has been investigated the energetic spectrum of the intrinsic surface states of quasi-particle, originated in the dielectric nanoparticle $\mathcal{E}<<1$, and its dependence on radius $a$ of nanoparticle at the conditions, when the polarization interaction between the charge carrier and spherical interface of two mediums, plays dominant role. It is shown, that spectrum of the intrinsic surface states of quasi-particle with increasing the radius a of nanoparticle in such way that

$$
S=\left(a / b_{p(e, h)}\right)>>n^{2}
$$

transforms in the spectrum of Coulomb type

$$
E_{n l}(S)=-\frac{9}{4 n^{2}}+\frac{L^{2}}{S^{2}}
$$

where $n, l$ - principal and orbital quantum numbers, $L^{2}=l(l+1)$. Here the next energetic unit is used $(R y / 36)=\left(\hbar^{2} / 2 m_{p(e, h)} b_{p(e, h)}^{2}\right)$.

In the region of frequencies $\omega_{n l}(S)=\left(E_{n l}(S) / \hbar\right)$, corresponding to Coulomb states $(n, l)$ (4) of charge carriers in the nanoparticle with radius $S$ (3), the length of light wave is much bigger than sizes of those states $\left(\approx a_{p(e, h)}(1)\right)$. So, the behavior of such Coulomb states in the electromagnetic field can be described in framework of dipole approach [9-11].

To evaluate the value of dipole moment $D_{1 s}^{2 p}(S)$ it is enough to consider the transition between lowest Coulomb states (4), for example between ground $|1 s\rangle=(n=1, l=0)$ and $\mid 2 p>=(n=2, l=1)$ Coulomb states. The transition between such states is allowed by selection rules in Coulomb field (the principal quantum number $n$ may change ad arbitrium and orbital quantum number $l$ may change per unit). The expression for dipole moment of transition in the nanoparticle with radius $S$ can be written as

$$
D_{1 s}^{2 p}(S)=e \Lambda|1 s| r|2 p\rangle \approx 2^{13 / 2} \cdot 3^{-4} \Lambda \alpha^{-1} e b_{p(e, h)}
$$

This expression is valid if the radius of nanoparticle $S$ obeys the inequality ( 3 ) and condition

$$
\alpha S>1
$$

In Eq. (5) $|1 s\rangle$ and $|2 p\rangle$ are the wave functions of Coulomb states $1 \mathrm{~s}$ - and $2 \mathrm{p}$ - correspondingly, $\Lambda$ and $\alpha$ are determined by expressions:

$$
\begin{aligned}
& \Lambda=\left(3 \varepsilon_{1} /\left(2 \varepsilon_{1}+\varepsilon_{2}\right)\right) \\
& \alpha=(9 / 4)\left(m_{0} / m_{p(e, h)}\right)
\end{aligned}
$$

(where $\mathrm{m}_{0}$ - mass of electron in vacuum).

The force of oscillator of transition for charge carrier with effective mass $m_{p(e, h)}$ from ground 1 s state to $2 \mathrm{p}$ state is known [11]:

$$
f_{1 s}^{2 p}(a)=\frac{2 m_{p(e, h)}}{\hbar e^{2}}\left[\omega_{2,1}(a)-\omega_{1,0}(a)\right]\left|D_{1 s}^{2 p}\right|^{2}
$$

where

$$
\hbar \omega_{2,1}(a)=E_{2,1}(a) \text { and } \hbar \omega_{1,0}(a)=E_{1,0}(a)
$$

the energy of Coulomb levels $2 p-$ and $1 \mathrm{~s}$ correspondingly. Using Eq. (4), Eq. (5) and Eq. (7), the force of oscillator of transition (8) can be written in form:

$$
f_{1 s}^{2 p} \approx\left(2^{9} \cdot 3^{-5}\right) \Lambda^{2} \alpha^{-2}
$$

As the transition $|1 s\rangle \rightarrow|2 p\rangle$ between Coulomb states (4) occurs in the nanoparticles with large radius $S$ (at the same time the radius $S$ should obey the conditions (3) and (6)) we can in Eq. (5) and Eq. (6), in first convergence by parameter $\left((\alpha S)^{-1} \ll 1\right)$, neglect by dependence on radius of nanoparticle $S$.

\section{The Absorption and Scattering of Light on Coulomb States of Nanoparticles}

The cross section of absorption of light on spherical nanoparticle with radius $a$ can be express via its polarizability $A^{\prime \prime}(\omega, a)$ [11,13,]:

$$
\sigma_{a b s}(\omega, a)=4 \pi(\omega / c) A^{\prime \prime}(\omega, a)
$$

where $\omega$ - the frequency of external electromagnetic field 
$c$ - the velocity of light in vacuum. At temperatures

$$
T<\left(E_{b} / k\right)
$$

less than binding energy

$$
E_{b}=\left|E_{n l}(S \rightarrow \infty)\right| \approx\left(8,51 \cdot 10^{-1}\right) n^{-2}(\mathrm{eV})
$$

of Coulomb states $(n, l)$ (4) (where $k$ - Boltzmann constant), the polarizability of charge nanoparticle can be found by considering the nanoparticle as single giant ion $[10,11]$. The main contribution in polarizability $A^{\prime \prime}(\omega, a)$ comes from the transitions in discrete spectrum of such Coulomb states [10,11]. Keeping in $A^{\prime \prime}(\omega, a)$ the contribution from the one resonance term, corresponded to the transition between ground Coulomb states $1 \mathrm{~s}-$ and $2 \mathrm{p}$ , the polarizability $A^{\prime \prime}(\omega, a)$ of nanoparticle can be written as

$$
A^{\prime \prime}(\omega, a)=\frac{e^{2}}{m_{p(e, h)}} \cdot \frac{f_{1 s}^{2 p}}{\omega_{2,1}^{2}(a)-\omega^{2}-i \omega \Gamma_{2,1}(a)}
$$

where $\Gamma_{2,1}(a)$ - width of Coulomb $2 \mathrm{p}$ - level.

If we assume, that frequency of light wave $\boldsymbol{\omega}$ is far from the resonance frequency $\omega_{2,1}(a)$ (4) of Coulomb state $2 \mathrm{p}$ as well as widening $\Gamma_{2,1}(a)$ of level $2 \mathrm{p}$ is small $\left(\Gamma_{2,1}(a) / \omega_{2,1}(a)<<1[14]\right)$, we can qualitatively evaluate the polarizability $A^{\prime \prime}(\omega, a)$ (13) of nanoparticle. Taking into account (4) we have

$$
A^{\prime \prime}(\omega) \approx\left(2^{7} \cdot 3^{-1}\right) \varepsilon_{2} \beta^{-1} f_{1 s}^{2 p} b^{3}
$$

The expression for elastic scattering cross-section for electromagnetic wave of frequency $\boldsymbol{\omega}$ on dielectric nanopticle with radius $S(3),(6)[11,13]$ is:

$$
\sigma_{s c}(\omega)=2^{7} \cdot 3^{-1} \pi^{3}(\omega / c)^{4}\left|A^{\prime \prime}(\omega)\right|^{2}
$$

\section{The Comparison Theory and Experiment}

In [14] the energetic spectrum of lowest Coulomb states $(n, 1)(4)$ of positron, localized in spherical nanoporous with radius $a \approx(15 \div 20) \mathrm{nm}$, being in nanocrystal metals $\mathrm{Al}, \mathrm{Cu}, \mathrm{Mo}, \mathrm{Pd}, \mathrm{Fe}$ and $\mathrm{Ni}$ [15] is investigated. It is found by experiment, that in porous silica, containing nanoporous with radius $a \approx(10 \div 30) \mathrm{nm}$ there exist the localized states of electrons [16]. The studied here intrinsic surface Coulomb states of quasi-particle, localized in spherical nanoporous with radius $a$ (3), (6), being in the nanocrystal metals and silica, are possible in processes of absorption (and radiation) at transitions $\left(n^{\prime}, l^{\prime}\right) \rightarrow(n, l)$ with frequencies

$$
\omega_{n, l}^{n^{\prime}, l^{\prime}}(a)=\left|E_{n^{\prime}, l^{\prime}}(a)-E_{n, l}(a)\right| / \hbar,
$$

according to formulas (4) and (12).

Let give an example of estimations of cross sections of absorption $\sigma_{a b s}(\omega, a)(10)$ and scattering $\sigma_{s c}(\omega, a)(15)$ of light on mentioned Coulomb states of positron in the nanopore of nanocrystal metals [14] as well as electron in the nanopore of silica [12] in case of preffered transition $(|1 s\rangle \rightarrow|2 p\rangle)$ at the experiment conditions $[15,16]$.

In the table we present the estimations of forces of oscillator of transitions $f_{1 s}^{2 p}$ (9), dipole moments of transitions $D_{1 s}^{2 p}(5)$, polarizabilities $A^{\prime \prime}(\omega, a)$ (14), cross sections $\sigma_{a b s}(\omega, a)$ (10) of light wave with frequency $\omega$ (at the same time the relation $\left(\omega / \omega_{2,1}(a)\right)^{2}=10^{-2}$ is hold and frequency of wave $\omega$ is in the infrared region) of the mentioned earlier Coulomb states. If we take into account (Table.1) that forces of oscillators of transitions $f_{1 s}^{2 p} \approx(0,94 ; 0,87)$ as well as dipole moments of transitions $D_{1 s}^{2 p} \approx(2,4 ; 2,7) D_{0} \quad$ (where $D_{0}=e \AA$ [Debye] $)$ in the nanoporous with radiuses $a \approx(10 \div 20)$ nm reaches the giant values (at two orders higher than typical values of forces of oscillators and dipole moments of transitions for semiconductors $A_{3} B_{5}$ and metals [1,911,14]) and dipole transitions in electromagnetic field between the nearest Coloumb levels $E_{n l}(a)$ (4) in the nanoporous are allowed by selection rules when principal quantum number $n$ is constant or change and orbital quantum number $l$ is changed per unit [1,9-11], then it becomes clearly that the considered in this paper quasizero-dimensional systems are strongly non-linear

\begin{tabular}{|c|c|c|c|c|c|c|c|c|c|c|}
\hline${ }^{\text {Matrix }}\left(\varepsilon_{1}\right)$ & $\begin{array}{c}\text { Nanopore } \\
\left(\varepsilon_{2}\right)\end{array}$ & $\Lambda$ & $\mathbf{m}_{\mathrm{p}(\mathrm{e})} / \mathbf{m}_{0}$ & $\alpha$ & $\beta$ & b, (Á) & $D_{1 s}^{2 p}{ }^{\left(\mathbf{D}_{\mathbf{0}}\right)}$ & $f_{1 s}^{2 p^{10^{-1}}}$ & $\begin{array}{c}A^{\mathrm{m}} \\
\left(10^{-24} \mathrm{~cm}^{3}\right)\end{array}$ & $\begin{array}{c}\sigma_{a b s} \\
\left(10^{-24} \mathrm{~cm}^{2}\right)\end{array}$ \\
\hline Metal $(\infty)$ & (1) & 1,5 & 1,0 & 2,25 & 1 & 3,18 & 2,37 & 9,4 & $1,310^{3}$ & $1,610^{7}$ \\
\hline Si $(11,7)$ & (1) & 1,44 & 1,0 & 2,25 & 0,84 & 3,77 & 2,7 & 8,67 & $2,3510^{3}$ & $2,9610^{7}$ \\
\hline
\end{tabular}
nanostructures for infra-red radiation.

Table 1. Parameters of bound states of positrons and electrons, localized in the nanoporous with permittivity $\varepsilon_{2}$ in the metal and semiconductor matrixes with permittivity $\varepsilon_{1}$. Here $a=(15-30) n m$-radius of nano-particle, $m_{p(e)}$ - effective mass of positron $($ electron $),\left(\right.$ ratio $\left.\left(\omega / \omega_{2,1}\right)^{2}=10^{-2}\right), f_{1 s}^{2 p}-$ the forces of oscilator of transition, $D_{1 s}^{2 p}$ - dipole moments of transition expressed in units $\left(D_{0}=e \dot{A}\right)$, [Debye], $A^{\prime \prime}$ - polarizability of nanoparticle, $\sigma_{a b s}-$ cross section of absorption of light by nanoparticle. 
From the estimations cited in the table, it follows, that the value of cross section of absorption of light in the nanoporous with radiuses $a \approx(10-30) \mathrm{nm}$ reaches giant values $\sigma_{a b s}(\omega, a) \approx 10^{-17}$ sm2. At the same time the $\sigma_{a b s}(\omega, a)$ is on seven orders higher than the typical values for atom cross sections of absorption [1, 9-11]. As the values of cross sections of scattering of light $\sigma_{a b s}(\omega, a)(10)$ in comparing with corresponding values of cross sections of absorption $\sigma_{a b s}(\omega, a)$ (10) in the experiment conditions $[15,16]$ are neglible small, then the values of $\sigma_{s c}(\omega, a)$ are omitted in the table.

\section{Conclusion}

So, the conclusion is that giant values of cross sections of absorption of light in the studied nanosystems enable to use the such nano-structures as new strong absorbent in the broad range of infra-red waves with lengths which depend on the nature of contact materials.

The new method which we used here (in contrast to the complicated and technically difficult quantm-mechanical methods [16]) enables to determine the analytical depens of the optical characteristics of the nano-systems (the forces of the oscillators and the forces of the dipole moments transitions, absorption cross-section and scattering of the light) on parameters of the nano-systems (radius nanoparticles, effective masses of the quasi-particles, permittivities of the nanoparticles and the surrounding matrixes).

It is important to note, as is shown in the presented article the quasi-zero-dimensional nano-systems, consisting from the dielectric nano-particles (nanoporous) of the spherical shape in the matrixes of the metal and semiconductors are strongly-absorbing nano-materials, namely for the infra-red radiation. The nano-systems, studied here, can be used as strongly-absorbing nanomaterials in the broad range of the infra-red waves for the constructing the artificial analog of the photosynthesis nano-structures.

\section{References}

[1] S.I.Pokutnyi .Phys.Express. 1 (2011) 158.

[2] S.I.Pokutnyi. J.Nanoscienc.Lett. 1 (2011) 191.

[3] S.I.Pokutnyi. Phys. Express.2 (2012) 20.

[4] S.I.Pokutnyi. Semiconductor. 46 (2012) 174.

[5] S.I.Pokutnyi.Tech.Phys.Lett. 39 (2013) 233.

[6] S.I.Pokutnyi. Semiconductor. 47 (2013) 128.

[7] S.I.Pokutnyi. J. Appl. Phys. 96 (2004) 11115.

[8] S.I.Pokutnyi. Phys. Lett.A. 342 (2005) 347.

[9] S.I.Pokutnyi. Solid State Phys. 39 (1997) 720.

[10] S.I.Pokutnyi. Solid State Phys. 39 (1997) 606.

[11] I.Pokutnyi. S.Semiconductor. 40 (2006) 223.

[12] S.I. Pokutnyi. Phys. Stat. Sol.(b). 165 (1991) 109.

[13] V. Gaitler, Quantum Theory of Radiation (Oxford U. P., New York, 1954)

[14] A.P.Shpak, S.I.Pokutnyi. Metal Phys. Advanc. Technol. 32 (2010) 859

[15] H.E.Shaefer. Nanostruct. Mater. 6 (1995) 869.

[16] J.I.Alferov. Progr. Phys.Sciens. 172 (2010) 1068. 